\title{
MAШAS PROTÉSICAS CON DISPOSITIVO EN LA CORRECCIÓN DEL PROLAPSO GENITAL
}

\author{
Vicente Solà D. ${ }^{1}$, Jack Pardo S. ${ }^{1}$, Marcelo Bianchi P. ${ }^{2}$, Paolo Ricci A. ${ }^{1}$, Enrique Guiloff F. ${ }^{1}$ \\ ${ }^{1}$ Unidad de Uroginecología y Cirugía Vaginal, ${ }^{2}$ Unidad de Climaterio, Clínica Las Condes.
}

\begin{abstract}
RESUMEN
Objetivo: Evaluar la factibilidad, seguridad y eficacia de la corrección quirúrgica del prolapso genital, con sistema Gynecare Prolift. Método: Durante junio y agosto del 2006, se realizó corrección de prolapso genital anterior y/o posterior en tres pacientes de la Unidad de Uroginecología y Cirugía Vaginal de Clínica Las Condes. Correspondían a prolapsos grado 2 y 4. Resultados: Se logró una corrección exitosa en los tres casos, no hubo complicaciones intraoperatorias, ni en el postoperatorio inmediato o tardío. El tiempo operatorio fue de 55 y 60 minutos para los dos casos de corrección posterior y de 90 minutos para el de anterior y posterior. La escala visual análoga del dolor a las 12, 24 y 48 horas, fue de puntuación 3. Todas expresaron su entera satisfacción frente a los resultados logrados. Conclusiones: La corrección quirúrgica del prolapso genital con sistema Gynecare Prolift, es factible, seguro y eficaz. Sólo el seguimiento a largo plazo permitirá objetivar la permanencia de estos excelentes resultados.
\end{abstract}

\section{PALABRAS CLAVES: Prolapso genital, malla protésica}

\section{SUMMARY}

Objective: To evaluate the feasibility, efficacy and safety of the genital prolapse surgery correction with Gynecare Prolift System. Method: Between June and August 2006, 3 cases of anterior and/or posterior genital prolapse with this new technique were performed, in patients of Urogynecology and Vaginal Surgery Unit of Las Condes Clinic. The prolapse degree was 2 and 4. Results: The correction was obtained in all cases. Intraoperative, immediate and remote postoperative period not registered any complication. The operating time was 55 and 60 minutes for the posterior correction and 90 minutes for anterior and posterior correction. The pain visual analogue scale at 12, 24 and 48 hours was 3 . All patients expressed total satisfaction with the surgical results. Conclusions: The surgical correction of the genital prolapse with system Gynecare Prolift is feasible, safe and effective. Only the long term following will allow observing the permanence of these excellent results.

\section{KEY WORDS: Genital prolapse, prosthetic mesh}

\section{INTRODUCCIÓN}

El prolapso genital es una de las patologías que frecuentemente afecta la calidad de vida de la mujer. Se presenta en el $50 \%$ de las multíparas (1) y su incidencia aumenta con la edad (2). Olsen en 1997 (3) publicó que el $11,1 \%$ de las mujeres que alcanzaban los 80 años habían sido operadas por prolapso y/o incontinencia urinaria. Al utilizar técnicas clásicas, el $29,2 \%$ de ellas presentaba recidiva, requiriendo una reoperación. Estos resultados han llevado a buscar nuevas técnicas quirúrgicas para evitar el fracaso de la corrección quirúrgica. Si entendemos el prolapso 
genital como una hernia (4), podemos reparar el defecto y reforzarlo con mallas, aplicando el mismo concepto de reparación de éstas como por ejemplo las incisionales, entre otras (5). Para ello, durante los últimos años se han confeccionado mallas más flexibles y de mayor maleabilidad, como lo son las de polipropileno, macroporo y monofilamento. Con estas nuevas mallas se ha logrado reducir la recurrencia del prolapso a un $3 \%(5,6)$.

Con el objetivo de revisar la factibilidad, seguridad y eficacia de la corrección del prolapso genital, con sistema Gynecare Prolift, se presenta nuestra experiencia preliminar con los primeros tres casos.

\section{MATERIAL Y MÉTODO}

Se realizó reparación de prolapso de pared vaginal anterior $\mathrm{y} / \mathrm{o}$ posterior, en tres pacientes (Tabla I) que requerían corrección quirúrgica, en la Unidad de Uroginecología y Cirugía Vaginal de Clínica Las Condes. Para ello se utilizó el nuevo sistema de reparación del suelo pélvico, Gynecare Prolift (Johnson \& Johnson, Somerville, New Jersey) (Figura 1). El estudio previo consistió en la clasificación del prolapso de acuerdo a la estandarización internacional de POP-Q. En aquellos casos que presentaban incontinencia de orina de esfuerzo o potencial, se realizó urodinamia con cistomanometría monocanal, medición de la presión de retro-resistencia uretral (URP) y Q-Tip Test. En dos pacientes se preparó la mucosa vaginal en forma previa a la cirugía con estriol local por 15 días, debido a que presentaban atrofia. Todas firmaron un consentimiento informado después de explicarles los detalles de la técnica quirúrgica. Se utilizó profilaxis antibiótica con cefazolina y metronidazol en tres dosis, iniciándola durante la cirugía. Durante el postoperatorio se utilizó analgésicos/antiinflamatorio por vía oral (ibuprofeno de $600 \mathrm{mg}$ cada 12 horas o ketoprofeno de $50 \mathrm{mg}$ cada 8 horas) y se aplicó la escala visual análoga del dolor a las 12, 24 y 48 horas.

\section{Caso 1}

54 años, multípara de 3 partos vaginales. Diagnóstico preoperatorio: Incontinencia de orina grado II + III (hipermovilidad uretral + deficiencia intrínseca esfinteriana) y rectocele grado 2 según clasificación POP-Q. Esta paciente además presentaba al examen físico gran debilitamiento del tabique recto-vaginal. El Q-Tip Test mostró hipermovilidad uretral. Además tenía gran dificultad evacuatoria de heces, requiriendo extracción manual. Cirugía planificada: TVT retropubiano (7), cistoscopia de revisión intraoperatoria, reparación de rectocele con sistema Prolift posterior y reparación de prolapso de nivel III de DeLancey.

\section{Caso 2}

77 años, multípara de 3 partos vaginales. Antecedente artrodesis de cadera derecha en 1966. Histerectomía total abdominal con conservación de los ovarios en 1977 por miomas sintomáticos. Posteriormente a esta cirugía presentó prolapso de cúpula vaginal por lo que se realizó sacrocolpopexia con malla de Prolene por vía abdominal en 2001, con fracaso posterior. Durante el 2001 cirugía por oclusión intestinal. Durante el 2005 y primer trimestre de 2006, mastectomía y radioterapia por cáncer de mama derecho. Vida sexual activa, con frecuencia cada 15 días. Al ingreso a nuestra unidad se consta un prolapso de cúpula grado 4 (POP-Q) (Figura 2). La paciente no tenía historia de incontinencia de orina, sin embargo se decide realizar una urodinamia preoperatoria por la potencialidad de su aparición después de la corrección quirúrgica del prolapso severo. La urodinamia monocanal + URP mostró incontinencia de orina de esfuerzo tipo III. El Q-Tip Test evidenció una uretra fija. Se le explicó a la paciente el riesgo teórico de aparición de incontinencia después de la corrección quirúrgica. Cirugía planificada: reparación anterior y posterior con sistema Prolift total y reparación de prolapso del nivel III de DeLancey.

Tabla I

PACIENTES SOMETIDAS A CORRECCIÓN DE PROLAPSO GENITAL CON SISTEMA GYNECARE PROLIFT

\begin{tabular}{lccclclc}
\hline Paciente & Edad & Paridad & IMC & Prolapso & POP-Q & Prolift & Tiempo \\
\hline 1 & 54 & 3 vaginal & 28 & Rectocele & $2^{\circ}$ & Posterior & $55 \mathrm{~min}$ \\
2 & 77 & 3 vaginal & 23 & Cúpula & $4^{\circ}$ & Anterior & $99 \mathrm{~min}$ \\
3 & 58 & 3 vaginal & 27 & Rectocele & $2^{\underline{0}}$ & Posterior & $60 \mathrm{~min}$ \\
\hline
\end{tabular}




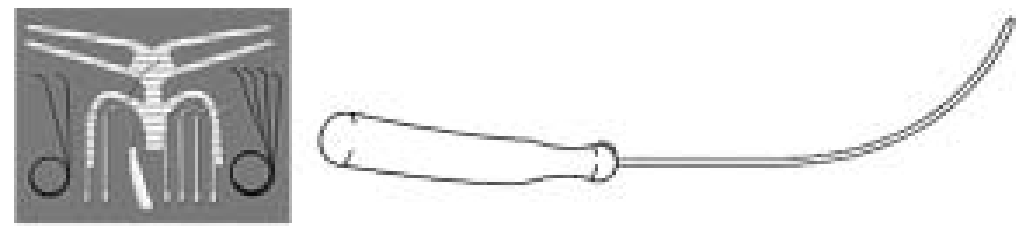

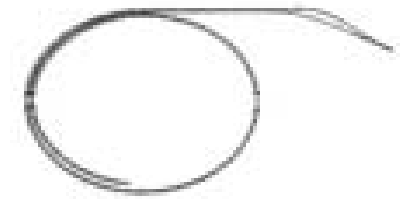

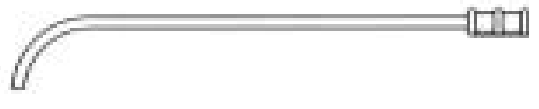

Figura 1. Set Gynecare Prolift. Sistema Gynecare Prolift, compuesto por Malla Gynemesh PS (polipropileno, macroporo, monofilamento), guía, cánula y dispositivo de tracción.

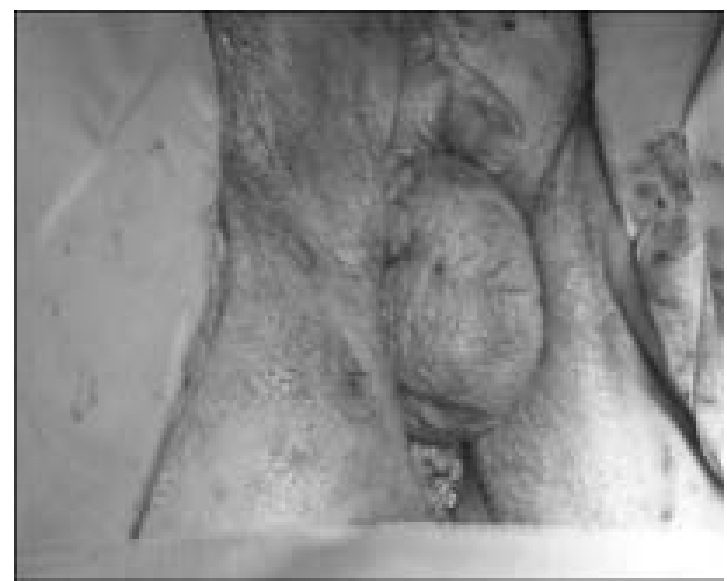

Figura 2. Prolapso de cúpula vaginal severo correspondiente al caso 2.

\section{Caso 3}

58 años, multípara de 3 partos vaginales. En tratamiento por hipertensión arterial, hipotiroidismo, esofagitis crónica distal, síndrome depresivo y dislipidemia. Además portadora de hígado graso. Al examen ginecológico se evidenció un rectocele grado 2 (POP-Q).

Descripción del sistema. Este sistema cuenta con implantes de malla y un set de instrumentos (una aguja con mango, cánulas y lazos) para facilitar su introducción y localización (Figura 1). MaIla: Se trata de una malla de polipropileno, macroporo, monofilamento, no absorbible (Gynecare Gynemesh PS). Según estas características, cumple con los requisitos de tipo I en la clasificación de mallas protésicas. Esta malla tiene la forma requerida para realizar una reparación vaginal total. Para ello cuenta con 6 prolongaciones o brazos: 4 para fijar la parte anterior del implante en el arco tendíneo de la fascia endopélvica (ATFE), utilizando el agujero obturador y 2 para fijar la parte posterior en el ligamento sacroespinoso por vía tranglútea. Las prolongaciones anteriores proximales poseen extremos cuadrados, mientras que las anteriores distales tienen extremos triangulares. Las prolongaciones posteriores tienen extremos redondeados (Figura 3). La malla para corrección total, permite recortarla para realizar si se requiere sólo una corrección anterior o una posterior (Figura 3). Guía: Se trata de un instrumento que permite formar la vía, a través de los tejidos, donde se fijarán las prolongaciones o brazos de los implantes de malla. Está formado por un mango y una prolongación con una curva ( $\mathrm{Fi}$ gura 1). Cánula: Instrumento que se introduce para crear el canal por donde pasan los brazos de la malla. Se coloca sobre la guía antes de pasarla y se deja en su lugar después de retirar la guía.

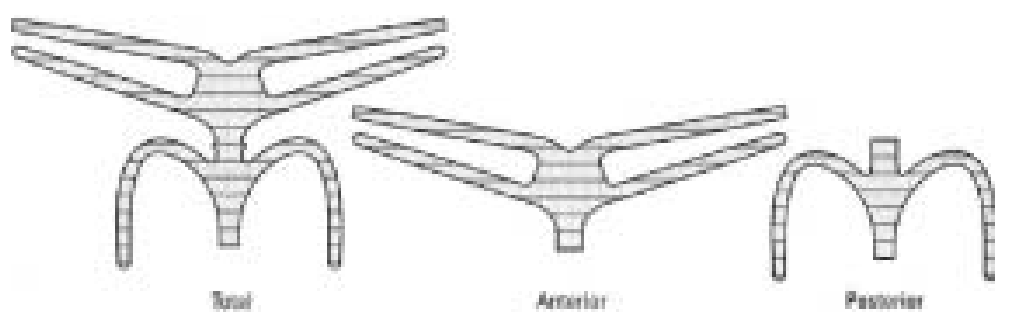

Figura 3. Malla del Sistema Gynecare Prolift. Malla Total para corrección de prolapso anterior y posterior, en casos con histerectomía total o cistocele y rectocele asociados. El corte de la malla total permite obtener una malla anterior y otra posterior. Malla anterior para corrección de cistoceles. Malla posterior para corrección de rectoceles. 
Permite el paso de los brazos de la malla y proteger el tejido adyacente (Figura 1). Dispositivo de tracción: Instrumento para facilitar la colocación y tracción de los brazos de la malla a través de las cánulas. El extremo distal posee un lazo que permite asegurarlo para evitar que se salga (Figura 1).

Uso del sistema Prolift: La cánula se ensambla en la aguja de la guía antes de introducirla a través de los puntos previamente marcados en la piel (Figura 4). Una vez situada la cánula se saca la guía (Figura 5). Luego se pasa el dispositivo de tracción a través de la cánula, se engancha 2,5 a $3 \mathrm{~cm}$ del brazo de la malla en el lazo de este dispositivo (Figura 6) y se tracciona para exteriorizarlos y así ajustar la malla.
Técnica quirúrgica específica: Se coloca la paciente en posición ginecológica, con flexión de muslo-abdominal de 90 grados. Vaciamiento de orina vesical con sonda Foley de 16 french. Sistema anterior: Se colocan 4 puntos tractores de labios mayores con menores. Colocación de pinzas Allice en línea media vaginal, desde la base del cuello vesical hasta un centímetro de la cúpula vaginal o fondo de saco anterior en el caso de existir cérvix. Infiltración submucosa vaginal con solución vasoconstrictora (epinefrina) en dilución 1 a 300.000 , en toda la extensión del prolapso. Incisión medial con bisturí en zonas traccionadas con pinzas Allis. Disección con gasa y tijera de Metzenbaum de toda la fascia vesico-vaginal, hasta la palpación de ambas espinas ciáticas y espa-
Figura 4. Preparación de la guía. Preparación de la guía con su cánula, antes de su introducción en la paciente.

Figura 5. Extracción de la guía dejando la cánula. Introducción de la guía y la cánula en la paciente, extracción de la guía dejando la cánula.

Figura 6. Paso del dispositivo de tracción y salida de los brazos de la malla por la cánula. Se pasa el dispositivo de tracción a través de la cánula y se engancha el brazo de la malla, 2 ó 3 centímetros, para luego traccionarlo y hacerlo pasar por la cánula al exterior.
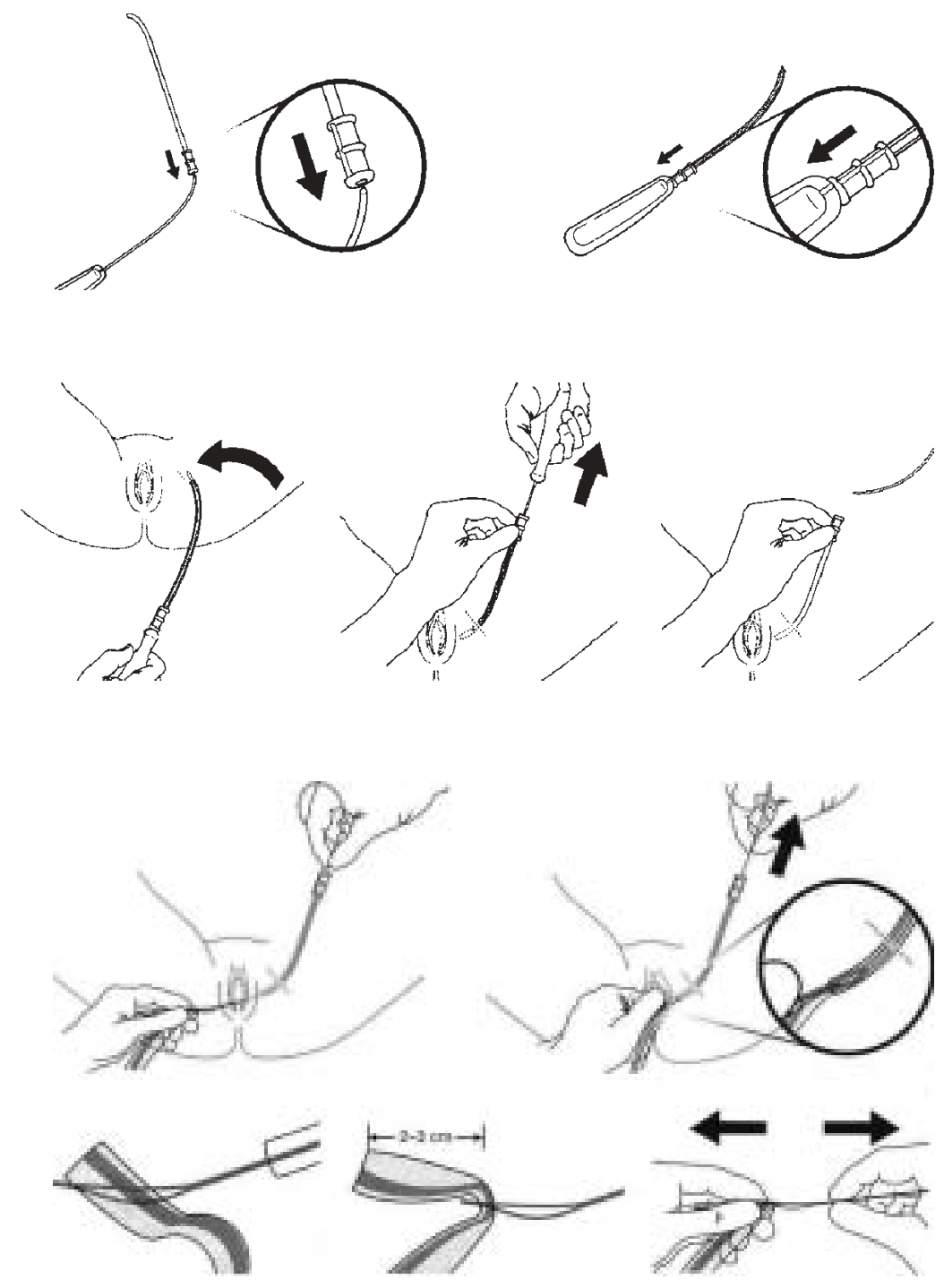
cios paravaginales. Se marca con lápiz en el cruce de línea que nace a nivel del meato urinario y corta con rama ascendente del pubis y borde de membrana obturatriz. Una segunda marca un centímetro hacia fuera y dos hacia abajo desde la primera, a ambos lados. Incisión de $4 \mathrm{~mm}$ con bisturí, en las marcas anteriores. Se coloca valva Breyski ancha para separar vejiga, se pasa aguja del dispositivo por la primera marca, pasando un centímetro distante del hueso pubiano a través del ATFE, de afuera hacia adentro. Se retira la aguja y se pasa el dispositivo de tracción por la cánula. Se pasa una segunda aguja en la segunda marca, también a través del ATFE, un centímetro proximal a espina ciática. Se repiten ambas maniobras al lado contralateral (Figuras 7, 8 y 9). Sistema posterior: Se marcan dos puntos tres centímetros por fuera y posterior al ano a cada lado. Colocación de pinzas Allis en línea media vaginal, desde inicio del rectocele hasta $1 \mathrm{~cm}$ de la cúpula vaginal o fondo de saco posterior en caso de tener cérvix. Infiltración submucosa vaginal con solución vasoconstrictora (epinefrina) en dilución 1 a 300.000 , en toda la extensión del prolapso. Incisión medial con bisturí en zonas traccionadas con pinzas Allis. Disección con gasa y tijera de Metzenbaum de toda la fascia recto-vaginal, hasta la palpación de ambas espinas ciáticas y espacios pararectales. Se coloca valva Breyski ancha para traccionar recto contralateralmente al paso de la aguja de la guía. Se pasa aguja por dicha marca atravesando la grasa perirectal y apoyado por el dedo índice del cirujano, se lleva ésta por debajo de la espina ciática y se atraviesa el ligamento sacroespinos 2 $\mathrm{cm}$ distales a la espina. Se visualiza la aguja y cánula, se retira la primera y se pasa el dispositivo

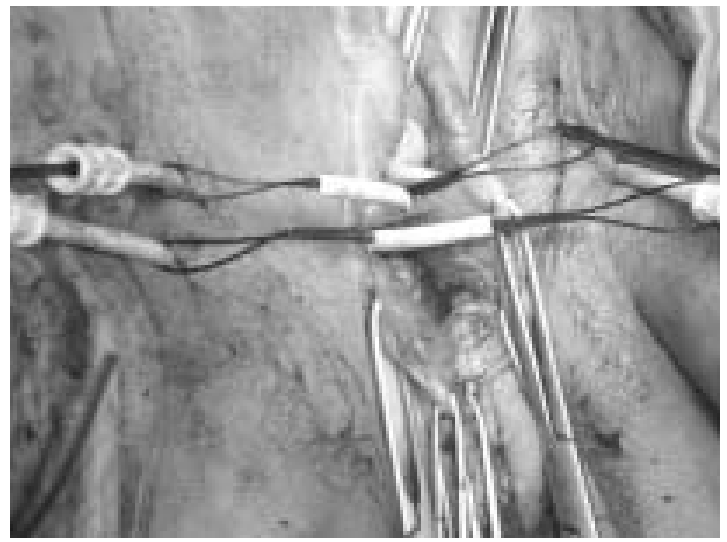

Figura 7. Cánulas con los dispositivos de tracción. Cánulas con los dispositivos de tracción pasados y asegurados, en el caso de corrección anterior.

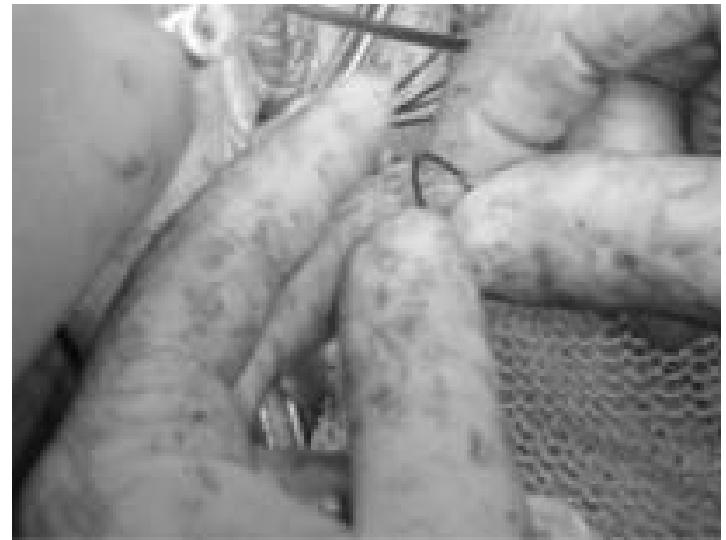

Figura 8. Paso del brazo de la malla al lazo del dispositivo de tracción. Se pasan 2 a 3 centímetros del brazo de la malla por el lazo del dispositivo de tracción.

de tracción, haciendo un reparo de ésta. Se repite la maniobra contralateralmente.

\section{RESULTADOS}

No se registraron complicaciones durante el intraoperatorio, el postoperatorio inmediato (hasta 7 días) o tardío (después de 7 días). El tiempo quirúrgico fue de 55 y 60 minutos para los dos casos de reparación posterior y de 90 minutos para la reparación total. En las tres pacientes se logró la corrección total del prolapso genital que motivó la corrección quirúrgica, objetivado mediante el examen ginecológico durante el postoperatorio. Las tres pacientes no relataron sentir dolor importante durante el postoperatorio, por lo que no requirieron analgésicos adicionales. El puntaje

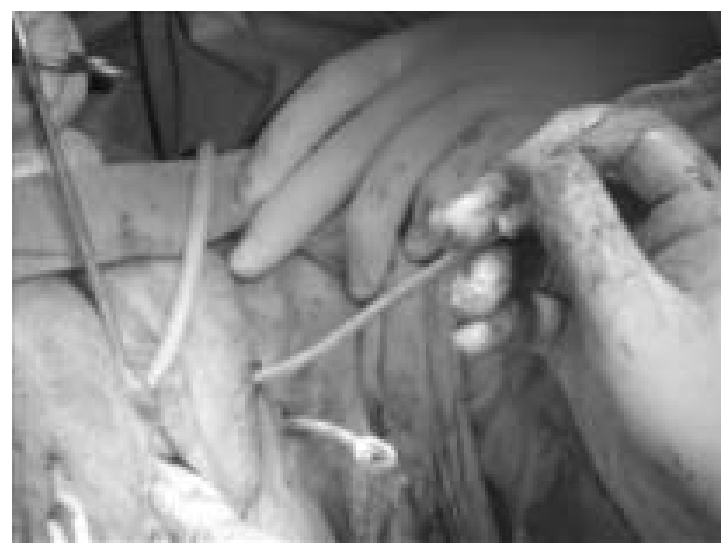

Figura 9. Ajuste de la malla. Una vez exteriorizados los brazos de la malla a través de la cánula, se traccionan para lograr el ajuste. 
en la escala visual análoga del dolor fue menor a 3. Al preguntarles por la satisfacción frente a los resultados, las tres manifestaron su entera satisfacción.

\section{DISCUSIÓN}

El sistema Prolift, a nuestro juicio, permite lograr una mayor tensión sobre los tejidos afectados por el defecto herniario. Esto se logra gracias al mayor ajuste y tracción que se realiza a través de los brazos que salen por las cánulas. En cambio con las técnicas de corrección con refuerzo de malla libre de tensión (5), no se logra este grado de refuerzo y tracción. Con Prolift se utilizan lugares seguros para la fijación de la malla, tal es el caso del arco tendíneo, fascia y membrana obturadora y el ligamento sacroespinoso.

$\mathrm{Si}$ la comparamos con técnicas como la sacrocolpopexia con malla (8) por vía vaginal, abdominal o laparoscópica, la aplicación de Prolift resulta mucho más fácil y con menor invasión. Por lo que teóricamente debería tener menos posibilidad de complicaciones, como las generadas por el anclaje a sacro.

Por otro lado se trata de una técnica que permite reforzar sin la necesidad de realizar suturas en la fascia endopélvica, no requiere laparotomía, permite reparar el defecto manteniendo una indemnidad del resto de la anatomía del suelo pélvico, por lo que podemos hablar de una cirugía mínimamente invasiva. No requiere resección de mucosa vaginal, por lo que no se acorta la vagina. Este beneficio queda especialmente demostrado en el segundo caso, en que se trataba de una paciente de 77 años, con vida sexual activa, con un prolapso genital severo, en que quedaba totalmente excluida la posibilidad de técnicas como la de Lefort. Con Prolift se recuperó la anatomía normal de la vagina.

Este sistema permite realizar cirugías de antiincontinencia, en forma concomitante, como ocurrió en el primer caso, en que se realizó un TVT con técnica clásica.

Importante nos parece recalcar el procedimiento de hidrodisección de la mucosa vaginal con el vasoconstrictor, lo que facilita la separación de la fascia, y además permite disminuir el sangrado que podría hacer más difícil este paso.

Otro tema importante a discutir es el tipo de malla utilizado por este sistema. El tipo de malla que se escoge para una cirugía de suelo pélvico no da lo mismo, ya que puede incrementar las potenciales complicaciones. Esto queda demostra- do por publicaciones como la de Julian (9), que demostró un éxito de $100 \%$ en la corrección de cistocele con malla versus $66 \%$ en casos controles sólo con colporrafia anterior. Pero utilizó Marlex, obteniendo un $25 \%$ de complicaciones relacionadas a esta versus $0 \%$ en los casos de colporrafia anterior, sin asociación a malla. Prolift utiliza una malla de polipropileno, macroporo, monofilamento. Se trata de una malla de prolene (Gynemesh PS) aprobada en mayo del 2003 por la FDA (Food and Drug Administration), que tiene mejores propiedades respecto a sus antecesoras. Es una malla denominada tipo I según la clasificación de materiales protésicos. Es una malla que teóricamente cumple mejor con la biointegración esperada en la reparación de defectos del suelo pélvico. No debemos olvidar que la malla protésica ideal es aquella que es biocompatible, inerte, estéril, no carcinogénica, que no cause reacciones alérgicas ni inflamatorias y resistente $(10,11)$. En este caso se trata de una malla macroporo, es decir mayor a 75 micrones. El tamaño del poro permite que la malla tenga una mayor flexibilidad (12), y además la infiltración de fibroblastos, paso de leucocitos, angiogénesis y mecanismos de anclaje o biointegración (13). Aquellas mallas con poros entre 50 y 200 micrones proporcionan mejor anclaje, ya que facilitan la infiltración de colágeno (14). Además estos poros permitirán el paso de macrófagos (50 micrones) y leucocitos, disminuyendo el riesgo de infecciones (bacterias $1 \mathrm{mi}-$ crón) (15). El riesgo de infección, además se reduce con la característica de ser monofilamento, lo que evitará la permanencia de bacterias en los pequeños espacios generados entre las fibras. Otro de los riesgos del uso de mallas es la erosión de pared vaginal, con posterior exteriorización. En nuestra experiencia en la corrección de cistocele y/o rectocele con malla Gynemesh PS, hemos aplicado un total de 110 mallas (anteriores, posteriores o combinadas), desde noviembre del 2004 hasta agosto del 2006, y hemos observado sólo una erosión $(0,9 \%)$. Se trataba de una paciente con hipotrofia de la mucosa vaginal, sin preparación previa, en la que se presentó una pequeña erosión, que no requirió extracción del trozo de malla. La primera parte de esta experiencia fue publicada durante 2005 (5), mientras que una segunda parte se encuentra actualmente en prensa.

Muchas de estas erosiones son el resultado de subinfecciones, por ello nuestro protocolo de trabajo contempla de rigor el uso de antibiótico (cefazolina + metronidazol en tres dosis), aún cuando teóricamente con las características de 
esta malla, por lo antes expuesto, se reduce la probabilidad de infección. Otra forma de reducir el riesgo de erosión es asegurando un buen tropismo de la mucosa vaginal, para ello si es necesario se puede preparar la mucosa previamente con estriol local. Por otro lado, se trata de una malla con bordes netos, cortados con láser, que además permite cortarla para utilizar sólo la parte de reparación anterior o la posterior, sin que pierda la elasticidad, forma o se desenrede.

Una vez finalizada la cirugía, es recomendable dejar gasas que compriman adecuadamente la mucosa vaginal, para evitar complicaciones como la acumulación de suero o sangre, las que pueden ser embebidas en furacin para evitar que se peguen.

Este sistema permite otorgar un buen refuerzo y solución en la reparación de prolapsos, en que se desea restaurar la anatomía, con una cirugía con características de mínima invasión, con una recuperación rápida; y sobre todo evitando durante esta reparación, dañar otras estructuras del suelo pélvico, permitiendo mantener el delicado equilibrio como queda expresado en la teoría integral de Ulmsten y Petros.

Si utilizamos malla en la reconstrucción del suelo pélvico, la malla que debemos elegir debe ser de polipropileno, macroporo y multifilamento, según todo lo expuesto anteriormente. Sin embargo, no es posible decir aún con el mismo apoyo teórico y científico-bibliográfico, cuándo debemos utilizar mallas y cuándo no (16); menos aún podemos responder actualmente cuándo ocupar la malla Gynemesh PS (que recortamos según el requerimiento) (5) y cuándo utilizaremos el sistema Gynecare Prolift. Es decir cuál es la mejor indicación para cada sistema de malla. A nuestro parecer este nuevo sistema debería utilizarse de preferencia en prolapsos severos, en cambio para los casos moderados en que se requiera malla, se podría escoger malla Gynemesh PS.

\section{CONCLUSIÓN}

La corrección quirúrgica del prolapso genital con sistema Gynecare Prolift, es factible, seguro y eficaz. Sólo el seguimiento a largo plazo permitirá objetivar la permanencia de estos excelentes resultados.

\section{BIBLIOGRAFÍA}

1. Carey MP, Dwyer PL. Genital prolapse: vaginal versus abdominal route of repair. Curr Opin Obstet Gynecol 2001;13(5):499-505.
2. MacLennan AH, Taylor AW, Wilson DH, Wilson D. The prevalence of pelvic floor disorders and their relationship to gender, age, parity and mode of delivery. BJOG 2000;107(12):1460-70.

3. Olsen AL, Smith VJ, Bergstrom JO, Colling JC, Clark $A L$. Epidemiology of surgically manager pelvis organ prolapse and urinary incontinence. Obstet Gynecol 1997;89(4):501-6.

4. Solà V, Pardo J, Ricci P, Guiloff E. Mallas en el tratamiento quirúrgico del prolapso genital en la mujer. Rev Med Clin Condes 2005;16(3):168-79.

5. Solà V, Pardo Schanz J, Ricci P, Guiloff E. Tension free monofilament polypropilylene mesh in cystocele and rectocele concomitant repair. Actas Urol Esp 2005;29(10):977-80.

6. De Tayrac R, Gervaise A, Chauveaud A, Fernandez $\mathrm{H}$. Tension-free polypropylene mesh for vaginal repair of anterior vaginal wall prolapse. J Reprod Med 2005;50(2):75-80.

7. Solà V, Pardo J, Ricci P, Guiloff E, Chiang H .TVT associated with other gynecological operations in the same procedure: results and complications. Arch Esp Urol 2005 58(10):983-8.

8. Solà V, Pardo J, Ricci P, Guiloff E, Alcalde JL, Tacla X. Cirugía mínimamente invasiva para el prolapso de cúpula vaginal: promontosuspensión con malla y anclaje helicoidal de titanio, a través de minilaparotomía con separador elástico de automantención. Rev Chil Obstet Ginecol 2005;70(6):364-8.

9. Julian TM. The efficacy of Marlex mesh in the repair of severe, recurrent vaginal prolapse of the anterior midvaginal wall. Am J Obstet Gynecol 1996;175(6): 1472-5.

10. Cumberland $\mathrm{VH}$. A preliminary report on the use of prefabricated nylon weave in the repair of ventral hernia. Med J Aust 1952;1(5):143-4.

11. Scales JT. Materials for hernia repair. Proc Royal Soc Med 1953;46:647-52.

12. Chu CC, Welch L. Characterization of morphologic and mechanical properties of surgical mesh fabrics. J Biomed Mater Res 1985;19(8):903-16.

13. Falconer $\mathrm{C}$, Soderberg M, Blomgren B, Ulmsten U. Influence of different sling materials on connective tissues metabolism in stress urinary incontinent women. Int Urogynecol J Pelvic Floor Dysfunct 2001;12 Suppl 2:S19-23.

14. Bobyn JD, Wilson GJ, MacGregor DC, Pilliar RM, Weatherly GC. Effect of pore size on the peel strength of attachment of fibrous tissue to poroussurfaced implants. J Biomed Mater Res 1982;16(5): 571-84.

15. Brun JL, Bordenave L, Lefebvref, Bareille R, Barbie $\mathrm{C}$, Rouaise F, Baquey $\mathrm{CH}$. Physical and biological characteristics of the main biomaterial used in pelvis surgery. Biomed Mater Eng 1992;2(4):203-25.

16. Davila GW. Introduction to the 2005 IUGA Grafts Roundtable. Int Urogynecol J Pelvic Floor Dysfunct 2006;17 Suppl 7:4-5. 